\title{
Spectrophotometric Determination of Amoxicillin Trihydrate by Coupling with Diazotized 2, 4-Dinitroaniline
}

\author{
Hisham Amin Taher Al-Herki ${ }^{1 *}$, Subhi Mohsin Jarullah Al-Mtwaiti ${ }^{2}$ \\ ${ }^{1,2}$ Department of Chemistry, College of Education for Pure Sciences, University of Mosul, Mosul, \\ Iraq
}

E-mail: 1 hisham.esp93@student.uomosul.edu.iq , 2 drsubhi74@uomosul.edu.iq

(Received June 05, 2021; Accepted July 27, 2021; Available online September 01, 2021)

DOI: 10.33899/edusj.2021.130393.1165, (c) 2021, College of Education for Pure Science, University of Mosul.

This is an open access article under the CC BY 4.0 license (http://creativecommons.org/licenses/by/4.0/)

\begin{abstract}
:
A simple, accurate and sensitive spectrophotometric method has been developed for the determination of amoxicillin trihydrate (AMOX) as pure and in pharmaceutical preparations (capsule). This method based on the coupling reaction of drug with diazotized 2,4- dinitroaniline reagent in an alkaline medium at $20^{\circ} \mathrm{C}$ to produce an intense red, water-soluble dye that is stable and has a maximum absorption at $555 \mathrm{~nm}$. Beer's law was obeyed over the concentration range 3-16 $\mu \mathrm{g} / \mathrm{ml}$ with molar absorptivity of $1.1 \times 10^{4} 1 . \mathrm{mol}^{-1} \cdot \mathrm{cm}^{-1}$. The limit of detection (LOD) is $0.1448 \mu \mathrm{g} / \mathrm{ml}$ while the limit of quantitation (LOQ) is $0.4825 \mu \mathrm{g} / \mathrm{ml}$. The method shows high accuracy (average recovery 100.43\%) and precision(relative standard division (RSD) is less than 1.4\%).The stoichiometry of the resulting azo dye has been also worked out and it is found to be 1:1 AMOX:Diazotized 2,4-Dinitroaniline.Standard addition method refers to the suggested method is free from interferences from common excipients. The developed method was successfully applied for the determination of studied drug in capsules comparable with the certified content value. .
\end{abstract}

Keywords: Spectrophotometry, Diazotization-coupling reaction, Amoxicillin Trihydrate, 2,4dinitroanilin

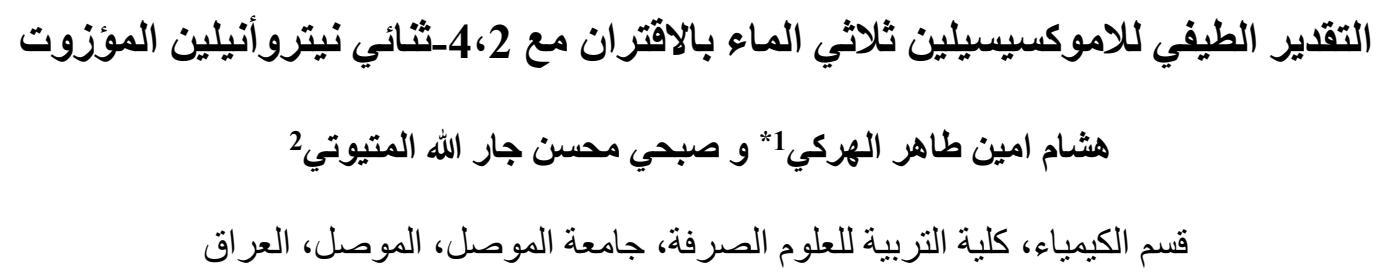


طريقة الاضافة القياسية متفقة على نحو جيد مع الطريقة المقترحة ضمن المدى المقبول للخطأ مما يدل على اند الطباء الطريقة ذات انتقائية

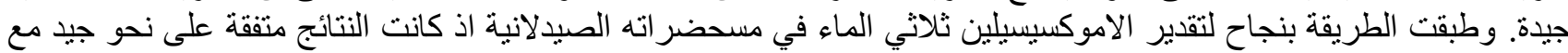

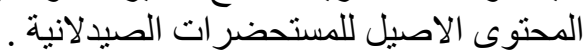

الكلمات المفتاحية : المطياف الضوئي ، تفاعل الاقتران الازوتي ، اموكسيسيلين ثلاثي الماء ، 2،4ثنائي نيترو انيلين المقدمة : المفات

الاموكسيسيلين ثلاثي الماء هو دواء شبه اصطناعي ينتمي الى فئة من المضادات الحيوية تسمى البنسيلينات ويستخدم لعلاج

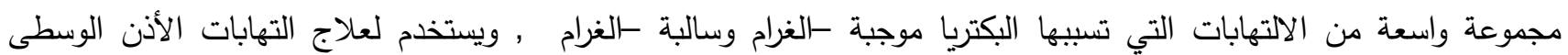

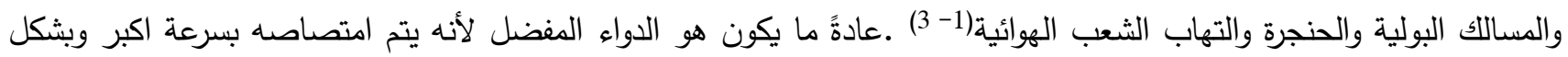

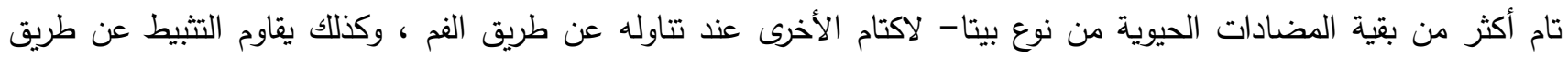

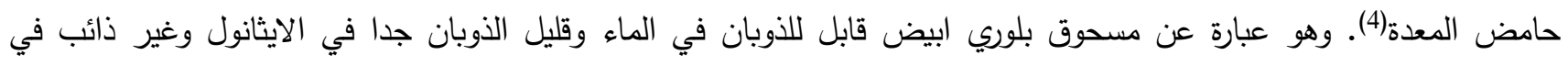

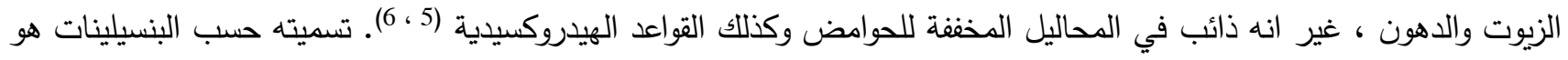
a-amino-p-hydroxy benzyl ك- 6-[D(-)-a-Amino-p-hydroxyphenyl acetamido]penicillanic acid 7-[2-Amino-2-(4-hydroxyphenyl)-acetyl]amino- (IUPAC) بينما تكون تسميته حسب نظام , penicillin 3,3,dimethyl-6-oxo-2-thia-5-azabicyclo [3,2,0] heptan-4-carboxylic acid

بالشكل (1) (7):<smiles>CC1(C)SC2C(NC(=O)C(N)c3ccc(O)cc3)C(=O)N2C1C(=O)O</smiles>

Formula structure: $\mathrm{C}_{16} \mathrm{H}_{19} \mathrm{~N}_{3} \mathrm{O}_{5} \mathrm{~S} \cdot 3 \mathrm{H}_{2} \mathrm{O}$

M.Wt: $419.449 \mathrm{gm} / \mathrm{mole}$

\section{Scheme 1 : Amoxicillin trihydrate structure}

استخدمت طرائق تحليلية مختلفة لتقدير دواء الاموكسيسيلين ثلاثي الماء وتتضمن : الطرائق الطيفية لتقدير كميات مايكروغرامية من المركب الدوائي بالاعتماد على تفاعلات الاقتران الازوي مع كواشف متتوعة( 9،9) ، تفاعلات تكوين التجمعات

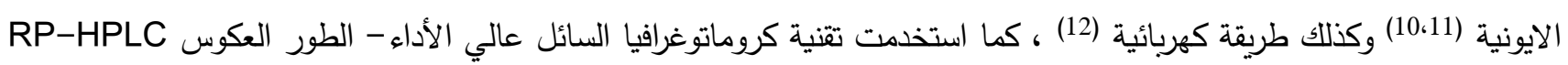
(13،14) ، كما استخدمت طريقة حركية (15) وتفاعل زرقة بروسيان (16) و تفاعلات تكوين معقدات الثحنة المنتقلة (17). ولقد طورت في هذا البحث طريقة طيفية لتقدير الاموكسيسيلين ثلاثي الماء بشكله النقي وفي مستحضراته الصيدلانية (كبسول) بالاعتماد على اقترانه مع الكاشف المؤزوت 4،2- ثنائي نيتروأنيلين في الوسط القاعدي ليعطي صبغة التئي ازوية ملونة ومستقرة لها اقصى امتصاص عند 555 نانوميتر لاعنى الجزء العملي: 


\section{الاجهزة المستعملة}

Double beam - spectrophotometer Shimadzu UV-1800 PC UV- استعمل جهاز المطياف الضوئي BS-11 Lab مع خلايا كوارتز ذات عرض 1سم ، كما تم تسخين المحاليل باستعمال حمام مائي من نوع لمعible

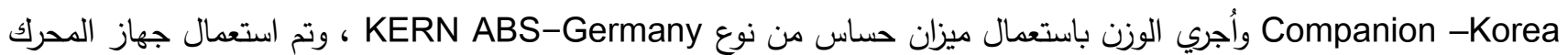
المغناطيسي نوع Elektro.mag لغرض اكمال الاذابة . المواد الكيميائية المستخدمة

كل المواد المستعملة في البحث كانت على درجة عالية من النقاوة ومصنعة من شركات عالمية معتمدة مثل شركة Fluka - $\mathrm{AlFa}, \mathrm{BHD}$, وتم الحصول على الاموكسيسيلين ثلاثي الماء من قبل شركة ادوية سامراء - العراق (SDI) . محلول اموكسيسيلين ثلاثي الماء القياسي (100 مايكروغرام/مللتر)

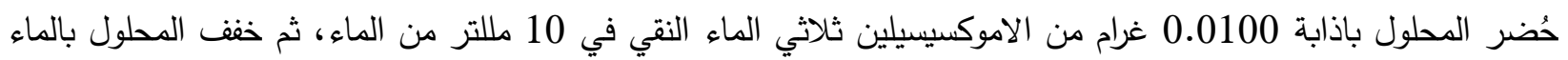
المقطر في قنينة حجمية سعة 100 مللتر . محلول 4,2-ثنائي نيترو أنيلين (0.1\%) حُضر المحلول باذابة 0.1000 غم من 4,2- ثنائي نيتروأنيلين (2,4-DNA) في 50 مللتر من الاسيتونتريل ,ثم اكمل الحجم بالماء المقطر الى حد العلامة في قنينة حجمية سعة 100 مللتر (18) (حيث انه غير ذائب في في الماء ) . محاليل الحوامض المعدنية (1 عياري ) حُضرت المحاليل بتخفيف الكمية المناسبة من كل حامض مركز في قنينة حجمية سعة 100 مللتر باضافتها الى الماء المقطر ثم التكملة بالماء المقطر الى حد العلامة .

محاليل القواعد ( 1 عياري ) حُضرت بإذابة الكمية المناسبة من كل قاعدة بالماء المقطر وأكمل الحجم إلى حد العلامة في قنينة حجمية سعة 100 مللتر •

محلول نتريت الصوديوم (1\%) حُضر بإذابة 1 غم من نتريت الصوديوم بالماء المقطر وأكمل الحجم إلى حد العلامة بالماء المقطر في قنينة حجمية سعة 100 مللتر مال

محلول حامض السلفاميك (22) حُضر بإذابة 2غم من حامض السلفاميك بالماء المقطر وأكمل الحجم إلى حد العلامة بالماء المقطر في قنينة حجمية سعة 100 مللتر .

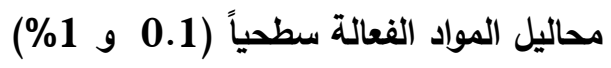
حُضرت المحاليل بإذابة 0.1000 غم من كل من الصوديوم الدوديكالية (SDS) وإذابة 1 غم من توين- 20 بالماء المقطر وأكمل الحجم إلى حد العلامة بالماء المقطر في قنانٍ حجمية سعة 100 مللتر. طريقة العمل والمنحني القياسي أُضيف 1.5 مللتر من الكاشف 2,4-DNA بتركيز 0.1\% الى سلسلة من القناني الحجمية سعة 25 مللتر , ومن ثم اضافة 0.5 مللتر من حامض الهيدروكلوريك (1عياري) تبعها اضافة 1 مللتر من نتريت الصوديوم (2\%) مع الرج لمدة 10 دقائق لاتمام

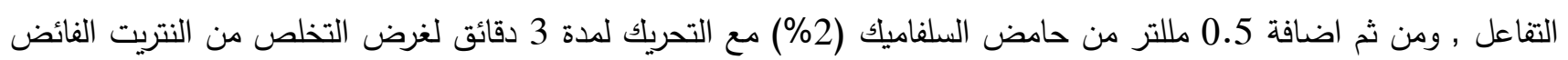


يتبعها اضافة حجوم متزايدة من الاموكسيسيلين ثلاثي الماء لتغطية المدى التقديري (3-16) مايكروغرام/مللتر ثم جعل الوسط

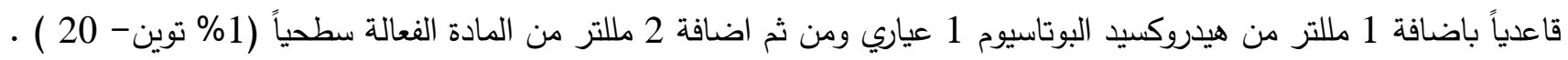

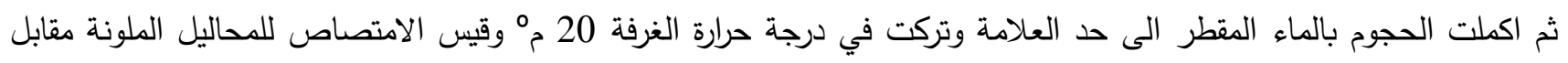
المحلول الصوري لها بعد 10 دقائق من التخفيف عند الطول الموجي 555 نانوميتر . تقدير الاموكسيسيلين ثلاثي الماء في مستحضراته الصيدلانية : الكبسول

تم وزن محتوى خمس كبسولات (تحتوي كل كبسولة على 500 ملغم من الاموكسيسيلين ثلاثي الماء ) وبعد مزجها جيداً وُزِن من المسحوق ما يكافئ 500 ملغم ( محتوى قرص دوائي واحد ) وتم اذابتها في 400 مللتر من الماء المقطر ثم تركت على ملى جهاز

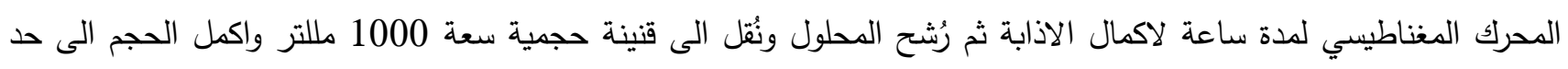
العلامة بالماء المقطر للحصول على 500 مايكروغرام/مللتر من محلول الكبسولات الدوائية , وحُضر منه محلول بتركيز 100 مايكروغرام/مللتر • وتم معاملتها وفق طريقة العمل المعتمدة.

النتائج و المناقشة مبدأ الطريقة تتم مفاعلة الكاشف 4,2-ثنائي نيتروأنيلين مع نتريت الصوديوم بوجود حامض الهيدروكلوريك لتكوين ملح الديازونيوم بحسب التبادي المعادلة الاتية(19):<smiles>CC(C)C(C)C</smiles><smiles>N#[N+]c1ccc([N+](=O)[O-])cc1[N+](=O)[O-]</smiles>

2, 4-Dinitroaniline Diazonium salt ثم يقترن الكاشف 4,2-ثنائي نيتروأنيلين المؤزوت مع الاموكسيسيلين ثلاثي الماء في الوسط القاعدي ليعطي صبغة ازوية لها اقصى امتصاص عند الطول الموجي 510 نانوميتر :<smiles>N#[N+]c1ccc([N+](=O)[O-])cc1[N+](=O)[O-]</smiles>

diazonium salt

Diazonium salt

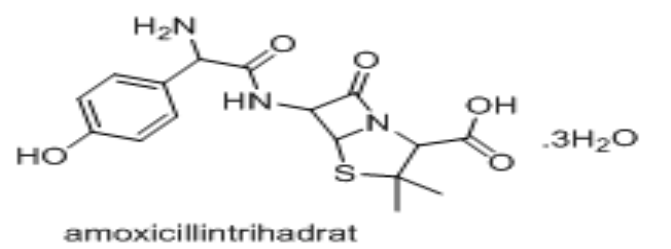

Amoxicillin Trihydrate

Red Azo dye 
درست مختلف المتغيرات المؤثرة على تفاعل تكوين الصبغة الازوية لغرض الحصول على الظروف المثلى لتكوينها باستخدام

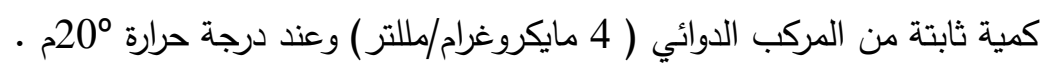
تأثير الكمية المثلى من الكاشف منف تم اختيار الكاشف 4,2-ثنائي نيترو أنيلين في هذا البحث لانه كاشف اقتران قوي لوجود مجموعتي نيترو ساحبة للالكترونات حيث تزيد من استقرارية الصبغة الناتجة بسبب حالة الرنين العالية . تمت دراسة تأثير تراكيز مختلفة من الكاشف (0.05 - 0.2\%) على امتصاص الصنبة الصغة المتكونة ـ وتشير النتائج في الجدولين 1و2 بان استخدام 1.5 مللتر من 0.1\% من الكاشف هي الكمية المثلى لاعطائها اعلى امتصاص. جدول (1) تأثير تركيز كاشف الاقتران على امتصاص الصبغة الناتجة

\begin{tabular}{|c|c|}
\hline $\begin{array}{c}\text { Concentration } \\
\text { of reagent }(\%)\end{array}$ & Absorbance \\
\hline 0.05 & 0.068 \\
\hline 0.1 & 0.112 \\
\hline 0.15 & Turbid \\
\hline 0.2 & Turbid \\
\hline
\end{tabular}

جدول(2) تأثير كمية الكاشف 42-ثنائي نيتروأنيلين على امتصاص الصبغة الناتجة.

\begin{tabular}{|c|c|}
\hline $\begin{array}{c}\text { Volume of } 0.1 \% \\
\text { of reagent }(\mathrm{ml})\end{array}$ & Absorbance \\
\hline 0.5 & 0.098 \\
\hline 1 & 0.114 \\
\hline 1.5 & 0.118 \\
\hline 2 & 0.099 \\
\hline
\end{tabular}

تأثير نوع الحامض على تكوين ملح الديازونيوم تم إجراء ازوتة الكاشف 4,2- ثنائي نيتروأنيلين في وسط حامضي باستخدام حوامض مختلفة (HCl و

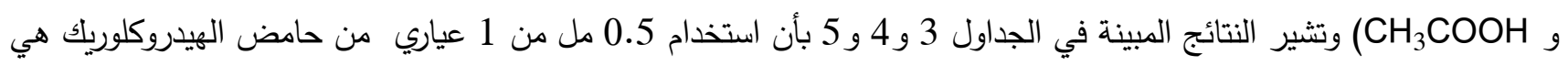
الكمية المثلى لتكوين ملح الدايزونيوم للكاشف 4,2-ثائي نيتروأنيلين 
جدول (3) تأثير نوع الحامض على امتصاص الصبغة المتكونة.

\begin{tabular}{|c|c|}
\hline $\begin{array}{c}\text { Type of acid } \\
(1 \mathrm{~N})\end{array}$ & Absorbance \\
\hline $\mathrm{HCl}$ & 0.116 \\
\hline $\mathrm{H}_{2} \mathrm{SO}_{4}$ & 0.097 \\
\hline $\mathrm{HNO}_{3}$ & 0.114 \\
\hline $\mathrm{CH}_{3} \mathrm{COOH}$ & 0.062 \\
\hline
\end{tabular}

جدول (4) تأثير تركيز حامض الهيدروكلوريك على امتصاص الصبغة المتكونة.

\begin{tabular}{|c|c|}
\hline $\begin{array}{c}\text { Normality of } \\
\mathrm{HCl}\end{array}$ & Absorbance \\
\hline 0.5 & 0.061 \\
\hline 1 & 0.117 \\
\hline 1.5 & 0.092 \\
\hline 2 & 0.014 \\
\hline
\end{tabular}

جدول (5) تأثير كمية حامض الهيدروكلوريك على امتصاص الصبغة المتكونة.

\begin{tabular}{|c|c|}
\hline $\begin{array}{c}\text { Volume of } 1 \mathrm{~N} \\
\mathrm{HCl}(\mathrm{ml})\end{array}$ & Absorbance \\
\hline 0.25 & 0.048 \\
\hline 0.5 & 0.118 \\
\hline 0.75 & 0.090 \\
\hline 1 & 0.018 \\
\hline
\end{tabular}

تأثير كمية النتريت

تم دراسة تأثير كمية النتريت اللازمة لازوتة الكاشف 4,2-ثنائي نيتروأنيلين وتبين ان الصبغة المتكونة تعطي اعلى شدة امتصاص عند استخدام 1 مل من 2\% من النتريت بزمن 10 دقائق لاتمام عملية الازوتة كما موضح في الجدولين(6و 7) . 
جدول(6) تأثير تركيز النتريت على شدة امتصاص الصبغة المتكونة.

\begin{tabular}{|c|c|}
\hline $\begin{array}{c}\text { Concentration } \% \\
\text { of nitrite }\end{array}$ & Absorbance \\
\hline 0.5 & 0.078 \\
\hline 1 & 0.117 \\
\hline 1.5 & 0.125 \\
\hline 2 & 0.128 \\
\hline 2.5 & 0.123 \\
\hline 3 & 0.108 \\
\hline
\end{tabular}

جدول (7) تأثير حجم نتريت الصوديوم بتركيز2\% على امتصاص الصبغة المتكونة.

\begin{tabular}{|c|c|}
\hline $\begin{array}{c}\text { Volume of } 2 \% \\
\mathrm{NaNO}_{2}(\mathrm{ml})\end{array}$ & Absorbance \\
\hline 0.5 & 0.091 \\
\hline 1 & 0.129 \\
\hline 1.5 & 0.114 \\
\hline 2 & 0.099 \\
\hline
\end{tabular}

تأثير تركيز حامض السلفاميك وكميته

من الضروري التخلص من حامض النتروز الغير متفاعل في الخطوة الاولى وذللك لاحتمالية حدوث تفاعل نيترة, وعليه يتم

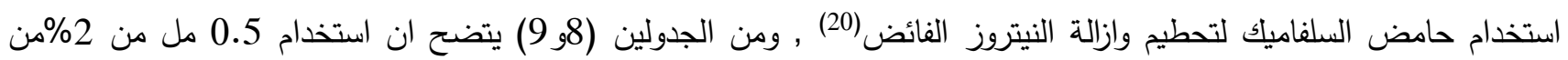
حامض السلفاميك وبزمن 3 دقائق هي الكمية المثلى له وحدوث تفاعل ازوتة ثابت . الجدول (8) تأثير تركيز حامض السلفاميك على امتصاص الصبغة المتكونة.

\begin{tabular}{|c|c|}
\hline $\begin{array}{c}\text { Concentration of } \\
\text { Sulphamic acid (\%) }\end{array}$ & Absorbance \\
\hline 1 & 0.113 \\
\hline 1.5 & 0.119 \\
\hline 2 & 0.126 \\
\hline 2.5 & 0.115 \\
\hline 3 & 0.112 \\
\hline
\end{tabular}


Journal of Education and Science (ISSN 1812-125X), Vol: 30, No: 4, 2021 (177-192)

جدول (9) تأثير كمية حامض السلفاميك على امتصاص الصبغة الناتجة.

\begin{tabular}{|c|c|}
\hline $\begin{array}{c}\text { Volum of } 2 \% \text { of } \\
\text { Sulphamic acid(ml) }\end{array}$ & Absorbance \\
\hline 0.25 & 0.124 \\
\hline 0.5 & 0.129 \\
\hline 0.75 & 0.122 \\
\hline 1 & 0.093 \\
\hline
\end{tabular}

تأثير نوع القاعدة وكميتها

ان تفاعل اقتران الكاشف المؤزوت 4,2-ثنائي نيتروأنيلين مع الاموكسيسيلين ثلاثي الماء يحدث في الوسط القاعدي , لهذا درس تأثير انواع مختلفة من القواعد ( $)$ و $\mathrm{NaOH}$ و $\mathrm{Na}_{2} \mathrm{CO}_{3}$ ) وتثير النتائج في الجداول10 و 11 و 12 أن استخدام 1 مل من 1 عياري من KOH هي افضل كمية لاعطائها اعلى امتصاص للصبغة الناتجة .

جدول(10) تأثير نوع القاعدة على امتصاص الصبغة المتكونة.

\begin{tabular}{|c|c|c|c|}
\hline $\begin{array}{c}\text { Type of base } \\
(1 \mathrm{~N})\end{array}$ & $\mathrm{NaOH}$ & $\mathrm{KOH}$ & $\mathrm{Na}_{2} \mathrm{CO}_{3}$ \\
\hline Absorbance & 0.115 & 0.126 & 0.022 \\
\hline
\end{tabular}

جدول(11) تأثير تركيز هيدركسيد البوتاسيوم على امتصاص الصبغة المتكونة.

\begin{tabular}{|c|c|}
\hline $\begin{array}{c}\text { Concentration of } \mathrm{KOH} \\
(\mathrm{N})\end{array}$ & Absorbance \\
\hline 0.5 & 0.033 \\
\hline 1 & 0.125 \\
\hline 1.5 & 0.091 \\
\hline 2 & 0.076 \\
\hline
\end{tabular}


جدول (12) تأثير كمية هيدروكسيد البوتاسيوم على امتصاص الصبغة المتكونة.

\begin{tabular}{|c|c|}
\hline $\begin{array}{c}\text { Volume of } 1 \mathrm{~N} \\
\mathrm{KOH}(\mathrm{ml})\end{array}$ & Absorbance \\
\hline 0.5 & 0.028 \\
\hline 1 & 0.127 \\
\hline 1.5 & 0.116 \\
\hline 2 & 0.104 \\
\hline
\end{tabular}

تأثير مواد الثد السطي دُرس تأثير انواع مختلفة من المواد الفعالة سطحياً الكاتيونية والانيونية والمتعادلة على امتصاص الصبغة المتكونة وكذلك

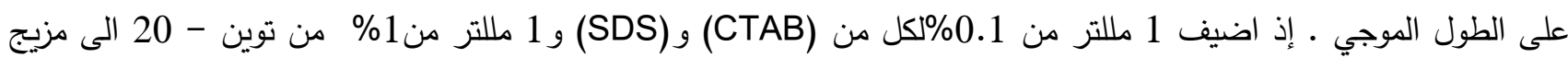
التفاعل و من ثم قياس الامتصاص للمحاليل عند الطول الموجي 510 نانوميتر , ومن ثم اجراء المسح لكل محلول لتحديد الطول الموجي الجديد اذ وجد أن استخدام توين - 20 المتعادل يعطي استقرار للصبغة الناتجة وكذلك زيادة في الطول الموجي من 510 الى 555 نانوميتر وزيادة في الامتصاص والتي اعتمدت في التجارب اللاحقة , كما تم دراسة تأثير كمية توين - 20 والتي كانت

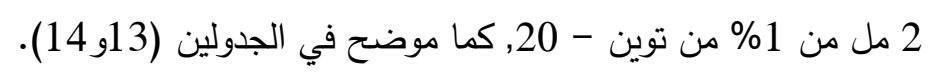

جدول (13) تأثير المواد الفعالة سطحياً على الامتصاص والتباين.

\begin{tabular}{|c|c|c|}
\hline \multirow{2}{*}{ Type of surfactant } & \multicolumn{2}{|c|}{ Absorbance } \\
\cline { 2 - 3 } & at $510 \mathrm{~nm}$ & at $\lambda$ max $(\mathrm{nm})$ \\
\hline Without & 0.131 & $0.131 / 510$ \\
\hline CTAB 0.1\% & 0.103 & $0.116 / 534$ \\
\hline SDS 0.1\% & 0.130 & $0.131 / 508.5$ \\
\hline Tween-20 1\% & 0.118 & $0.139 / 555$ \\
\hline
\end{tabular}

جدول (14) تأثير كمية 1\% من محلول توين - 20 على امتصاص الصبغة الناتجة.

\begin{tabular}{|c|c|}
\hline $\begin{array}{c}\text { Volume of } \begin{array}{c}\text { 1\% Tween-20 } \\
(\mathrm{ml})\end{array} \\
1\end{array}$ & Absorbance \\
\hline 1.5 & 0.138 \\
\hline 2 & 0.145 \\
\hline 2.5 & 0.149 \\
\hline
\end{tabular}


تأثير تسلسل الاضافة 2,4-DNA تمت دراسة تأثير تسلسل الاضافة على امتصاص الصبغة الناتجة وذلك باضافات متغيرة الترتيب للكاشف المؤزوت

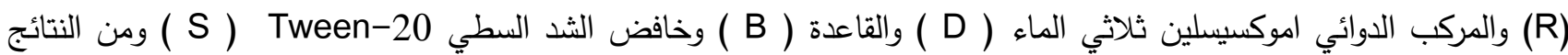
المبينة في الجدول(15) يتبين لنا ان التسلسل (ا) والمتبع في تثبيت الظروف المثلى اعطى اعلى امتصاص للصبغة المتكونة, لذا اعتمد هذا التسلسل في التجارب اللاحقة .

جدول(15) تأثير تسلسل الاضافة على امتصاص الصبغة المتكونة.

\begin{tabular}{|c|c|c|}
\hline $\begin{array}{c}\text { Order } \\
\text { number }\end{array}$ & Order of addition & Absorbance \\
\hline I & $\mathrm{R}+\mathrm{D}+\mathrm{B}+\mathrm{S}$ & 0.148 \\
\hline II & $\mathrm{R}+\mathrm{D}+\mathrm{S}+\mathrm{B}$ & 0.143 \\
\hline III & $\mathrm{R}+\mathrm{S}+\mathrm{D}+\mathrm{B}$ & 0.146 \\
\hline IV & $\mathrm{R}+\mathrm{B}+\mathrm{S}+\mathrm{D}$ & ---- \\
\hline
\end{tabular}

تأثير درجة الحرارة وزمن تكوين الصبغة الازوية

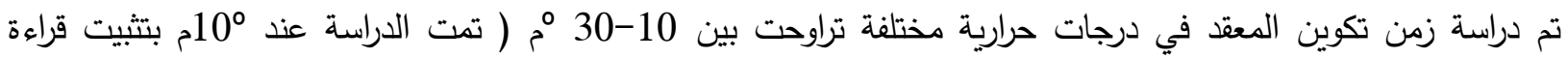
الحمام المائي عند 10 ووضع قطع من الثلج فيه من اجل الحفاظ على درجة الحرارة عند 10م) ، حيث وجد ان زمن تكوين الصبغة هو 10 دقائق وبزمن استقرار 50 دقيقة عند درجة حرارة الغرفة (20 مم). والتي استخدمت في الدراسات اللاحقة كما هو مبين في الجدول (16).

جدول (16) تأثير درجة الحرارة على زمن تكوين الناتج الملون واستقراره

\begin{tabular}{|c|c|c|c|}
\hline \multirow{2}{*}{$\begin{array}{l}\text { Standing time } \\
\text { min }\end{array}$} & \multicolumn{3}{|c|}{ Absorbance/ Temperature ${ }^{\circ} \mathrm{C}$} \\
\hline & $10^{\circ} \mathrm{C}$ & $\begin{array}{c}\text { Room Temp } \\
20^{\circ} \mathrm{C}\end{array}$ & $30^{\circ} \mathrm{C}$ \\
\hline After addition & 0.134 & 0.145 & 0.130 \\
\hline 5 & 0.134 & 0.145 & 0.130 \\
\hline 10 & 0.136 & 0.148 & 0.126 \\
\hline 15 & 0.136 & 0.148 & 0.124 \\
\hline 20 & 0.138 & 0.150 & 0.123 \\
\hline 25 & 0.136 & 0.149 & 0.121 \\
\hline 30 & 0.135 & 0.149 & 0.120 \\
\hline 40 & 0.134 & 0.147 & 0.120 \\
\hline 50 & 0.133 & 0.145 & 0.116 \\
\hline 60 & 0.132 & 0.145 & 0.117 \\
\hline 70 & 0.131 & 0.141 & 0.118 \\
\hline 80 & 0.129 & 0.143 & 0.115 \\
\hline 90 & 0.128 & 0.139 & 0.114 \\
\hline 100 & 0.127 & 0.137 & 0.114 \\
\hline 110 & 0.127 & 0.135 & 0.117 \\
\hline 120 & 0.126 & 0.130 & 0.117 \\
\hline 130 & 0.126 & 0.131 & 0.114 \\
\hline 140 & 0.126 & 0.130 & 0.116 \\
\hline
\end{tabular}




\section{طيف الامتصاص النهائي}

أجريت دراسة طيف الامتصاص النهائي للصبغة الازوية الناتجة من تفاعل اموكسيسيلين ثلاثي الماء مع الكاشف المؤزوت

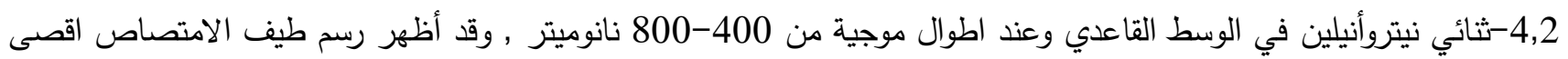
شدة امتصاص للصبغة عند طول موجي 555 نانوميتر مقابل محلولها الصوري , في حين تبين ان المحلول الصوري لله امتصاص

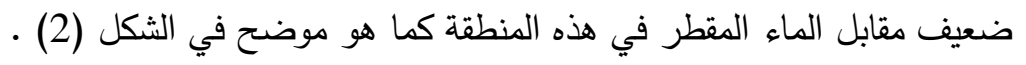
Absorbance sobance

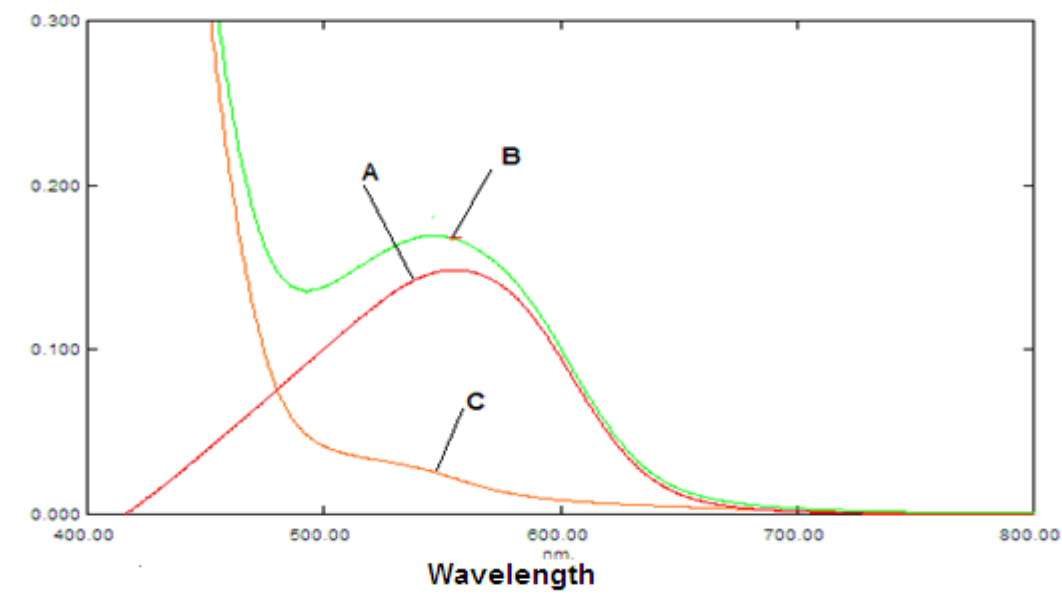

ين ثلاثي الماء مع الكاشف 4,2- مثنائي Wavelength $(\mathrm{nm})$

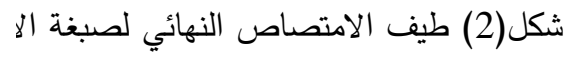
نيتروأنيلين المؤزوت في الوسط القاعدي. (A- طيف امنصاص الصبعه معابل المحلول الصوري , D- طيف امتصاص الصبغة مقابل الماء المقطر , C- طيف امتصاص المحلول الصوري مقابل الماء المقطر ) . التقدير الكمي أظهر رسم المنحني القياسي للامتصاص مقابل التراكيز المختلفة للاموكسيسيلين ثلاثي الماء ان الطريقة المقترحة تتبع قانون بير ضمن المدى 3-16 مايكروغرام/مللتر من المركب الدوائي مع انحراف سالب عن قانون بير بعد اعلى تركيز كما هو موضح

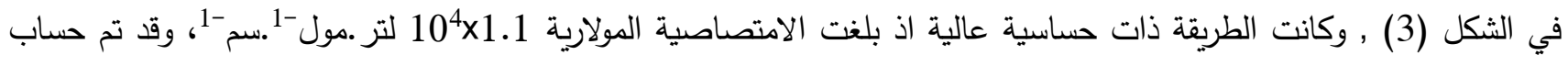
حد الكثف وكان 0.1464 مايكروغرام/مللتر وحد التقدير الكمي 0.4880 مايكروغرام/مللتر .

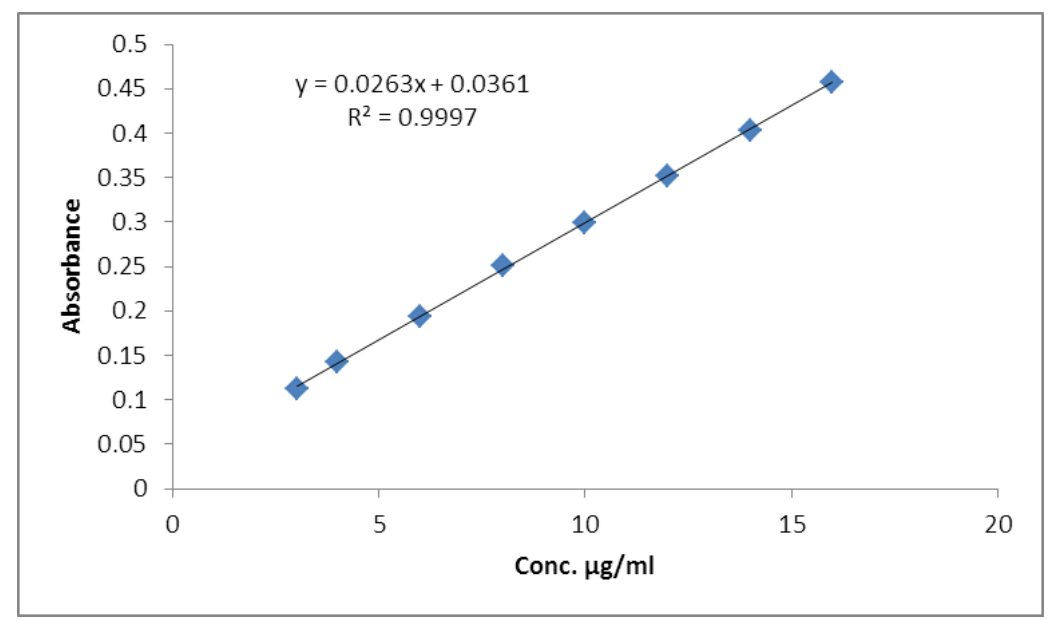

الثكل 3: المنحني القياسي لتقدير الاموكسيسيلين ثلاثي الماء 
دقة الطريقة وتوافقها تم استخدام ثلاثة تراكيز مختلفة من الاموكسيسيلين ثلاثي الماء لحساب الدقة والتوافق للطريقة المقترحة ـ وتثير النتائج في

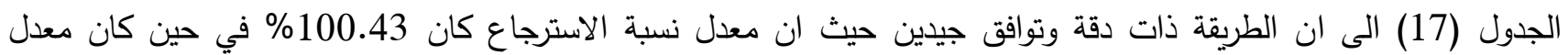

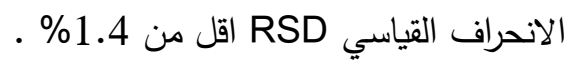
الجدول (17) دقة الطريقة وتوافقها لتقدير الاموكسيسيلين ثلاثي الماء

\begin{tabular}{|c|c|c|c|c|}
\hline \multicolumn{2}{|c|}{$\begin{array}{c}\text { Amount of Amoxicillin } \\
\text { trihydrate }(\mu \mathrm{g} / \mathrm{ml})\end{array}$} & \multirow{2}{*}{$\begin{array}{c}\text { Recovery } \\
(\%)\end{array}$} & $\begin{array}{c}\text { Average } \\
\text { Recovery*(\%) }\end{array}$ & $\begin{array}{r}\text { RSD * } \\
(\%)\end{array}$ \\
\cline { 1 - 2 } Taken & Found & & & 1.12 \\
\hline 4 & 4.11 & 102.75 & \multirow{2}{*}{100.43} & 1.33 \\
\hline 8 & 8.01 & 100.13 & & 0.89 \\
\hline 14 & 13.78 & 98.43 & & \\
\cline { 1 - 2 }
\end{tabular}

*Average of five determinations

الجدول (18) ملخص الخصائص الطيفية والبيانات الاحصائية للطريقة المقترحة

\begin{tabular}{|c|c|}
\hline $\begin{array}{c}\text { Limit of detection(LOD) } \\
(\mu \mathrm{g} / \mathrm{ml})^{*}\end{array}$ & 0.1448 \\
\hline $\begin{array}{c}\text { Limit of quantitation(LOQ) } \\
(\mu \mathrm{g} / \mathrm{ml})^{*}\end{array}$ & 0.4825 \\
\hline $\begin{array}{c}\text { Molar absorptivity } \\
\left(\mathrm{l} \mathrm{mol}^{-1} . \mathrm{cm}^{-1}\right)\end{array}$ & $1.1 \times 10^{4}$ \\
\hline Slope & 0.0263 \\
\hline Intercep & 0.0361 \\
\hline Determination coefficient $\left(\mathrm{R}^{2}\right)$ & 0.9997 \\
\hline
\end{tabular}

* For ten determinations of blank solution

طبيعة الصبغة المتكونة

درست طبيعة الصبغة الناتجة باستخدام طريقة التغيرات المستمرة (طريقة جوب Jop) (21), واشارت النتائج بان الصبغة

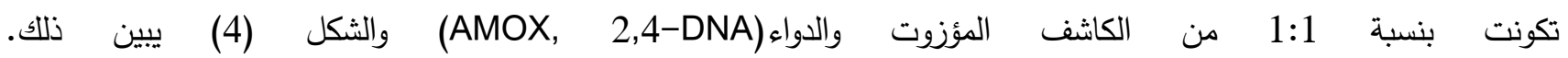




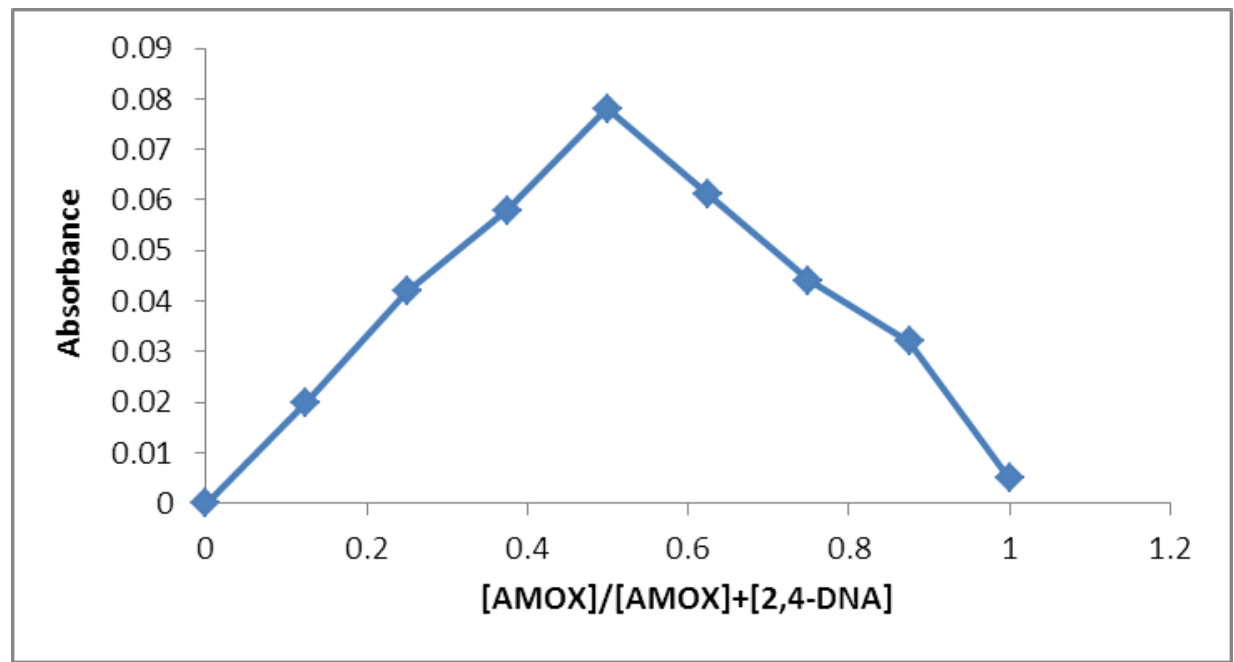

الثكل 4: طريقة جوب لـ 4,2-ثنائي نيتروأنيلين المؤزوت والمقترن مع الاموكسيسيلين ثلاثي الماء

بناءً على ما استتنج من هذه الدراسة فان الصيغة الكيميائية المقترحة للصبغة المتكونة من تفاعل الكاشف المؤزوت مع الاموكسيسيلين ثلاثي الماء ربما تكون بالثكل التالي :<smiles>CC1(C)SC2C(NC(=O)C(N)c3ccc(O)c(N=Nc4ccc([N+](=O)[O-])cc4[N+](=O)[O-])c3)C(=O)N2C1C(=O)O</smiles>

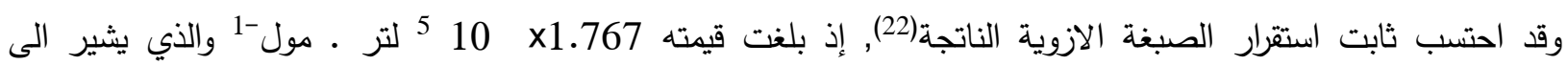
الاستقرارية العالية للصبغة الناتجة .

\section{تطبيق الطريقة على المستحضرات الصيدلانية}

طبقت الطريقة المقترحة لتقدير الاموكسيسيلين ثلاثي الماء في مستحضراته (الكبسول) ـ وتثير النتائج في الجدول (19) الى ان الطريقة ذات صلاحية تطبيق جيدة بالمقارنة مع المحتوى الاصيل للمستحضرات الصيدلانية المدروسة ـ كما تم فحص دقة الطريقة من خلال اختبار -t بمقارنة معدل الكمية المحسوبة $\mathbf{X}$ وانحرافها القياسيك Sع الكمية الحقيقة للدواء بر باستخدام المعادلة التالية(23): $t_{\exp }=\frac{|\mu-\bar{X}| x \sqrt{n}}{S}$

اذ تم حساب قيم texp للمستحضرات الصيدلانية للاموكسيسيلين ثلاثي الماء على شكل كبسول وقد اظهرت النتائج المبينة في الجدول(19) ان قيم t العملية عند مستوى ثقة 95\% ولاربع درجات حرية(خمس مكررات) اقل من قيمتها الجدولية(2.78) مما يدل على عدم وجود فرق معنوي بين القيمة الحقيقية والقيمة المقاسة للمركب الدوائي. 


\begin{tabular}{|c|c|c|c|c|c|c|c|}
\hline \multirow[t]{2}{*}{$\begin{array}{c}\text { Pharmaceutical } \\
\text { Preparation }\end{array}$} & \multirow[t]{2}{*}{$\begin{array}{c}\text { Certified } \\
\text { Value(mg) }\end{array}$} & \multicolumn{2}{|c|}{$\begin{array}{l}\text { Amount } \\
\text { Present } \\
(\mu \mathrm{g} / \mathrm{ml})\end{array}$} & \multirow{2}{*}{$\begin{array}{l}\text { Drug } \\
\text { Content } \\
\text { Found } \\
\text { (mg) }\end{array}$} & \multirow[t]{2}{*}{$\begin{array}{c}\text { Recovery } \\
\%\end{array}$} & \multirow[t]{2}{*}{$\begin{array}{c}\text { Average } \\
\text { Recovery } \\
\%\end{array}$} & \multirow[t]{2}{*}{ t-test } \\
\hline & & taken & found & & & & \\
\hline \multirow{3}{*}{$\begin{array}{l}\text { Amoxycillin } \\
\text { capsules } \\
\text { SDI-IRAQ }\end{array}$} & \multirow{3}{*}{500} & 4 & 4.03 & 503.75 & 100.75 & \multirow{3}{*}{100.72} & \multirow{3}{*}{2.19} \\
\hline & & 6 & 6.04 & 503.30 & 100.66 & & \\
\hline & & 8 & 8.06 & 503.75 & 100.75 & & \\
\hline \multirow{3}{*}{$\begin{array}{c}\text { Glomox } \\
\text { capsules } \\
\text { UAE }\end{array}$} & \multirow{3}{*}{500} & 4 & 4.11 & 513.75 & 102.75 & \multirow{3}{*}{101.09} & \multirow{3}{*}{2.75} \\
\hline & & 6 & 5.92 & 493.30 & 98.66 & & \\
\hline & & 8 & 8.15 & 509.35 & 101.87 & & \\
\hline
\end{tabular}

*Average of five determinations

\section{تقييم نتائج الطريقة المقترحة}

لاجل اثبات كفاءة الطريقة المقترحة لتقدير دواء الاموكسيسيلين ثلاثي الماء ونجاحها في التقدير بطريقة الازوتة وخلوها من تأثير

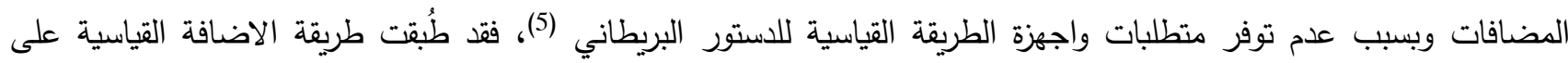
تركيزين مختلفين من كلا المستحضرين (AMOXYCILLIN) و (Glomox) كما في الشكل (5) و (6) ويُبين الجدول (20) ان طريقة الاضافة القياسية متفقة على نحو جيد مع الطريقة المقترحة ضمن المدى المقبول للخطأ مما يدل على ان الطريقة ذات انتقائية جيدة .

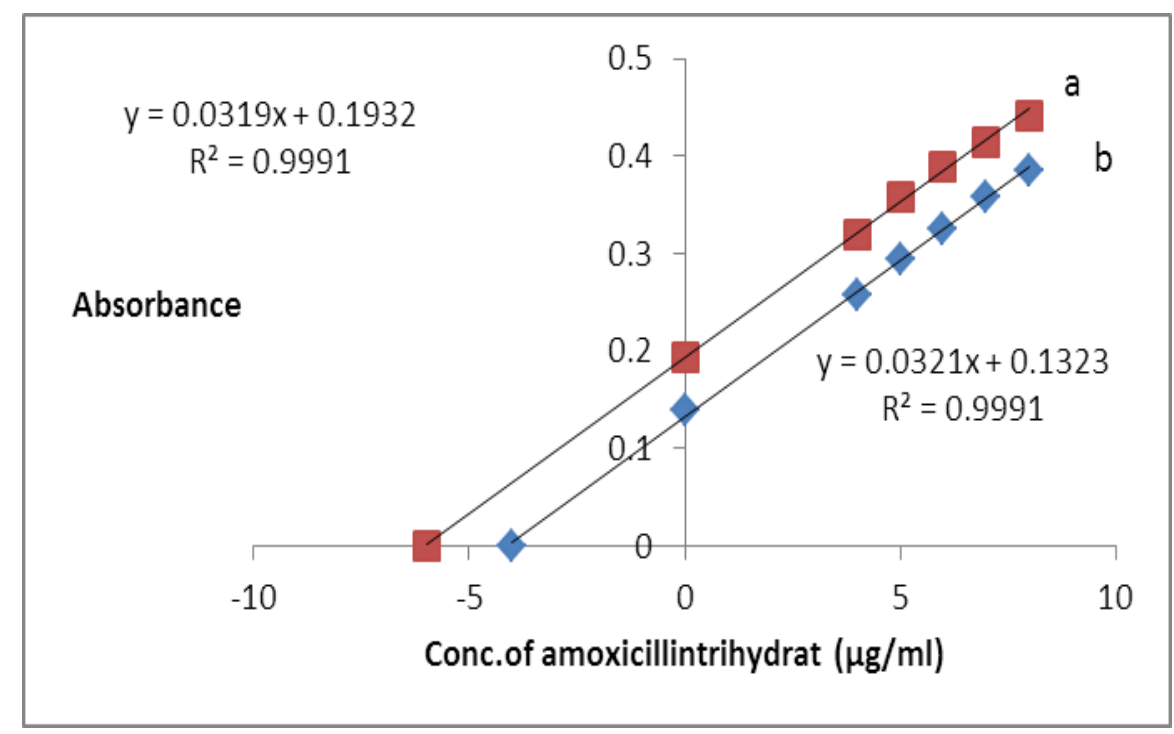

الثكل (5) منحني الاضافة القياسي لتقدير ( a = a a Amoxycillin المستحضر الصيدلاني 

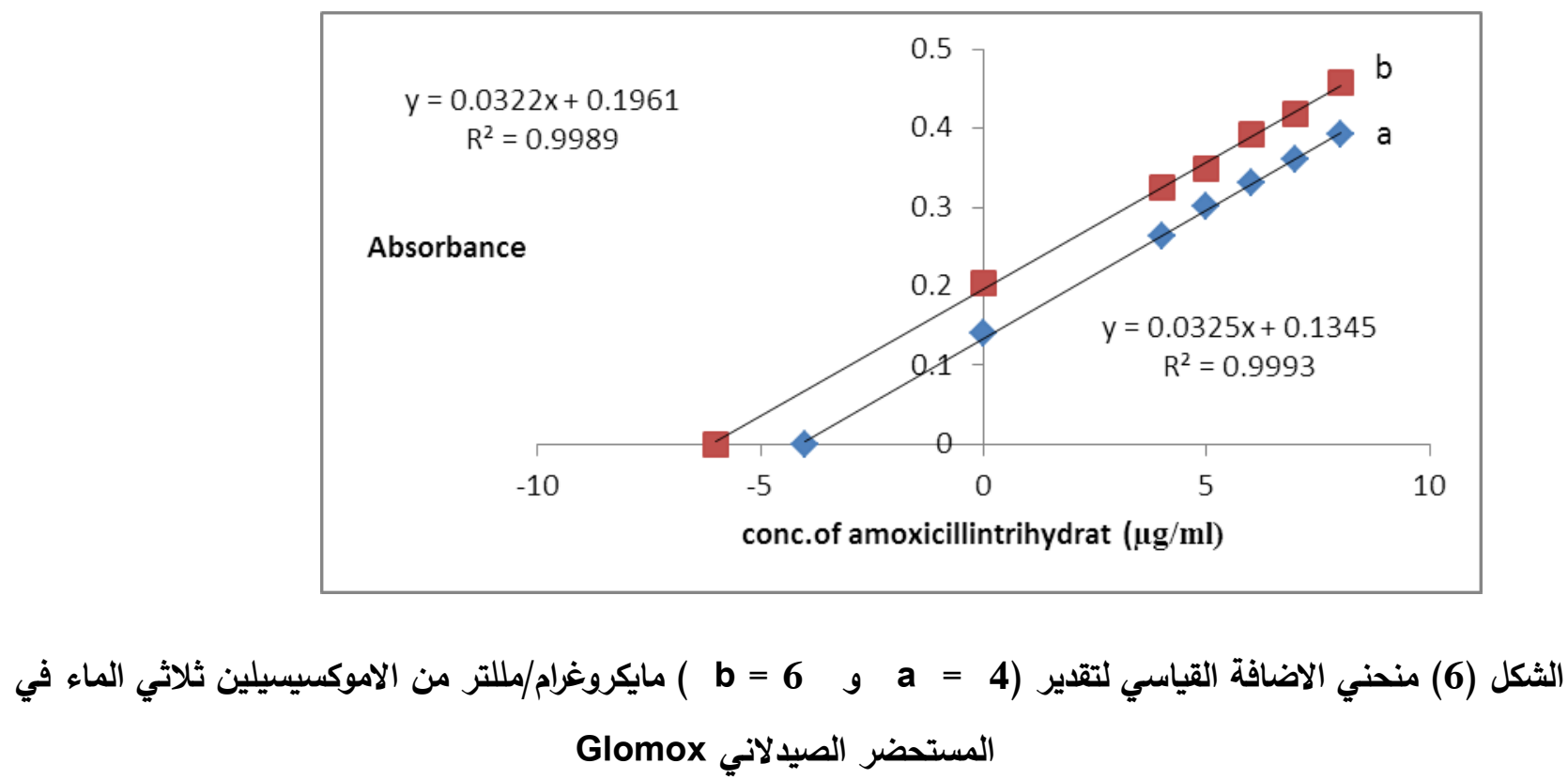

الجدول(20) تقدير الاموكسيسيلين ثلاثي الماء في المستحضرات الصيدلانية بطريقةالاضافة القياسية

\begin{tabular}{|c|c|c|c|c|}
\hline $\begin{array}{c}\text { Pharmaceutical } \\
\text { preparation }\end{array}$ & $\begin{array}{c}\text { Certified } \\
\text { Value(mg) }\end{array}$ & $\begin{array}{c}\text { Amount } \\
\text { Present } \\
(\mu \mathrm{g} / \mathrm{ml})\end{array}$ & $\begin{array}{c}\text { Drug } \\
\text { Content } \\
\text { Found(mg) }\end{array}$ & $\begin{array}{c}\text { Recovery } \\
(\%)\end{array}$ \\
\hline \multirow{2}{*}{$\begin{array}{l}\text { Amoxycillin } \\
\text { capsules }\end{array}$} & \multirow{2}{*}{500} & 4 & 515.20 & 103.04 \\
\hline & & 6 & 504.70 & 100.94 \\
\hline \multirow{2}{*}{$\begin{array}{l}\text { Glomox } \\
\text { capsules }\end{array}$} & \multirow{2}{*}{500} & 4 & 517.30 & 103.46 \\
\hline & & 6 & 507.50 & 101.50 \\
\hline
\end{tabular}

طورت طريقة طيفية حساسة وانتقائية لتقدير الاموكسيسيلين ثلاثي الماء تعتمد على اقتران الكاشف 2، 4-ثنائي نيتروأنيلين المؤزوت مع المركب الدوائي في الوسط القاعدي عند درجة حرارة 20 م لتكوين صبغة ازوية ملونة ذائبة في الماء ومستقرة وتعطي تلئي اقصى شدة امتصاص عند الطول الموجي 555 نانوميتر ، وطبقت الطريقة بنجاح لتقدير الاموكسيسيلين ثلاثي الماء في مسحضراته الصيدلانية اذ كانت النتائج متفقة على نحو جيد مع المحتوى الاصيل للمستحضرات الصيدلانية ومع نتائج طريقة

\section{References}

الاضافة القياسية للطريقة المقترحة .

1. Wilson O.C., Ole G. and Delgado N.J. (2004).Textbook of Organic Medicinal and Pharmaceutical Chemistry. $11^{\text {th }}$ Ebn, Lippincott Williams and Wilkns , 351 West Camden Street, Baltimore.MD21201, 310-317.

2. Broden RN. , Heel RC., Speight TM. and Avery CS. (1979.Amoxicillin injectable: a review of its antibacterial soectrum . pharmacokinetics and therapeutics use. Drugs, 18(3), 169-184.

3. Kagan .B, (1977). Ampicillin rash.Western J .of Medicine , 126(4), 333-335.

4. Toda P.A, Benfield P, Amoxicillin/clavulanic acidn; an ubdate of its antibacterial activity, pharmacokinetic properties and therapeutic use .Drugs; 1990. 39:264-307. 
5. British Pharmacopeia, (2013), CD-ROM, system simulation, the stationery office Ltd, London.

6. Sean. C, Sweet man, Martindale; The Complete Drug Reference, $33^{\text {rd }}$ edition, The Pharmaceutical press. UK, 2002, 149.

7. Maryadele .J.O, Neil P. (2006), The Merck Index; Encyclopedia of chemicals, $14^{\text {th }}$ edition, Merck Research Laboratories, USA, 92.

8. Othman. N and Al-Saffar S. R. (2017). "Spectrophotometric Determination of Amoxicillin by Coupling with Diazotized m-Nitroaniline", International Journal of Enhanced Research in Science, Technology and Engineering, 6(5):60-66.

9. Qader, H. A., and Fakhre, N. A. (2017). "Spectrophotometric determination of amoxicillin trihydrate in pure and pharmaceutical dosage forms". Ibn AL-Haitham Journal for Pure and Applied Science, 28(3), 142-153.

10. Antakli, S., Nejem, L., and Ahmad, W. A. (2019). "Determination of Amoxicillin Trihydrate by Analytical Spectrophotometry". Research Journal of Pharmacy and Technology, 12(6), 2716-2720.

11. Radhi, E. R. and Ali, K. J. (2019). Spectrophotometric Study for Comparison of Amoxicillin Trihydrate and Levofloxacin Hemihydrate Determination Using Bromocresol Green. International Journal of Pharmaceutical Research, 11(1):1107- 1113.

12. Kaur R., Kushwaha .J and Singh N., (2019)," Electro-Oxidation of Amoxicillin Trihydrate in Continuous Reactor by Ti/RuO 2 Anode". Sci, Total Environment , 677 : 84-97.

13. Hassouna M. and Mohamed M., (2018). "Development and Validation of RP-HPLC Method for Determination of Amoxicillin Residues and Application to NICOMAC Coating Machine". Journal of Analytical and Pharmaceutical Research, 7(5), 586-594.

14. Rajput, S., Bhamre, P., and Bambhrolia, S. (2014)., "RP-HPLC Method for Simultaneous Estimation of Ambroxol Hydrochloride, Potassium Clavulanate and Amoxicillin Trihydrate in Bulk Drugs and Laboratory Synthetic Mixture".J . Adv. Pharm. Edu and Res. 4(2):178185.

15. Karpova, S., and Ivashurr, M. (2014). "Quantitative Determination of Amoxicillin Trihydrate in Medical Forms Using Kinetic Method". Journal of Chemical and Pharmaceutical Research, 6(4), 1120-1125.

16. Farhadi .K, Ghadamgahi .S, Maleki, R .and Asgari .S, (2002)," Spectrophotometric Determination of Selected Antibiotics Using Prussian Blue Reaction", J. Chinese. Chem. Soci, 49: 993-997.

17. Amin, A. S., Moustafa, M. E., and Issa, Y. M. (1994). "Spectrophotometric Microdetermination of Ampicillin and Amoxicillin with Picric and Picramic Acids". Microchemical journal, 50(1), 6-13.

18. Saleem M. S., AL-Mtwaiti S.M. and AL-Ramadhani, T. S. (2013). "Spectrophotometric Determination of Salbutamol Sulphate by Coupling with Diazotized 2, 4-Dinitroaniline. Journal of Education and Science, 26(2), 54-64.

19. Panti M. and Neha, (2016)," Diazotization and Coupling Reaction of Differently Substituted Aromatic Amines and Investigation of Their Solvent-Chromic Behavior ", Der Chemica Sinica, 7(2): 93-100.

20.Clayden J., Greeves N., Warren S., and Wathers P.,(2001),"Organic Chemistry", $2^{\text {th }}$ Edn. Oxford uni. Press, London, pp.477.

21. Harvey D., (2000), "Modren Analytcal Chemistry", Mc Graw-Hill Higher Edu. USA.

22. Haris L.G (1988), "Analytical Chemistry ", Springer-Veralg, Berlin, Germany, P .67.

23. Skoog D.A., West D.M., Holler F.J. and Crouch S.R., (2004)," Fundamentals of Analytical of Analytical Chemistry", 8 Edition, Thomson Learning, USA. 